\title{
CONVERGENCE ANALYSIS OF THE JACOBI SPECTRAL-COLLOCATION METHODS FOR VOLTERRA INTEGRAL EQUATIONS WITH A WEAKLY SINGULAR KERNEL
}

\author{
YANPING CHEN AND TAO TANG
}

\begin{abstract}
In this paper, a Jacobi-collocation spectral method is developed for Volterra integral equations of the second kind with a weakly singular kernel. We use some function transformations and variable transformations to change the equation into a new Volterra integral equation defined on the standard interval $[-1,1]$, so that the solution of the new equation possesses better regularity and the Jacobi orthogonal polynomial theory can be applied conveniently. In order to obtain high-order accuracy for the approximation, the integral term in the resulting equation is approximated by using Jacobi spectral quadrature rules. The convergence analysis of this novel method is based on the Lebesgue constants corresponding to the Lagrange interpolation polynomials, polynomial approximation theory for orthogonal polynomials and operator theory. The spectral rate of convergence for the proposed method is established in the $L^{\infty}$-norm and the weighted $L^{2}$-norm. Numerical results are presented to demonstrate the effectiveness of the proposed method.
\end{abstract}

\section{INTRODUCTION}

In practical applications one frequently encounters the Volterra integral equations of the second kind with a weakly singular kernel of the form

$$
y(t)=g(t)+\int_{0}^{t}(t-s)^{-\mu} K(t, s) y(s) d s, \quad 0<\mu<1, \quad 0 \leq t \leq T,
$$

where the unknown function $y(t)$ is defined in $0 \leq t \leq T<\infty, g(t)$ is a given source function and $K(t, s)$ is a given kernel.

For any positive integer $m$, if $g$ and $K$ have continuous derivatives of order $m$, then from [5] there exists a function $Z=Z(t, v)$ possessing continuous derivatives of order $m$, such that the solution of (1.1) can be written as $y(t)=Z\left(t, t^{1-\mu}\right)$. As this will be the starting point of this paper, the detailed regularity result of (1.1) is given below.

Received by the editor March 24, 2008 and, in revised form, February 14, 2009.

2000 Mathematics Subject Classification. Primary 35Q99, 35R35, 65M12, 65M70.

The first author is supported by Guangdong Provincial "Zhujiang Scholar Award Project", National Science Foundation of China 10671163, the National Basic Research Program under the Grant 2005CB321703.

The second author is supported by Hong Kong Research Grant Council, Natural Science Foundation of China (G10729101), and Ministry of Education of China through a Changjiang Scholar Program.

(C)2009 American Mathematical Society Reverts to public domain 28 years from publication 
Lemma 1.1 ([5]). Assume that $g \in C^{m}(I)$ and $K \in C^{m}(I \times I)$ with $K(t, t) \neq 0$ on $I=[0, T]$. Then, the regularity of the unique solution of the weakly singular VIE (1.1) can be described by

$$
\begin{aligned}
& y \in C^{m}(0, T] \cap C(I), \quad \text { with } \quad\left|y^{\prime}(t)\right| \leq C_{\mu} t^{-\mu} \text { for } t \in(0, T] ; \\
& y(t)=\sum_{(j, k)_{\mu}} \gamma_{j, k}(\mu) t^{j+k(1-\mu)}+Y_{m}(t ; \mu), \quad t \in I,
\end{aligned}
$$

where $(j, k)_{\mu}:=\{(j, k): j, k$ are nonnegative integers, $j+k(1-\mu)<m\}, \gamma_{j, k}(\mu)$ are some constants, and $Y_{m}(\cdot ; \mu) \in C^{m}(I)$.

The above result implies that near $t=0$ the first derivative of the solution $y(t)$ behaves like $y^{(m)}(t) \sim t^{1-m-\mu}$, which indicates that $y \notin C^{m}([0, T])$. Several methods have been proposed to recover high-order convergence properties for (1.1) using collocation-type methods; see, e.g., [3, 4, 9, 15, 26, 27] and for the multi-step method, see, e.g., [17].

It is known that the singular behavior of the exact solution makes the direct application of the spectral methods difficult. To overcome this difficulty, we first apply the transformation

$$
\tilde{y}(t)=t^{\mu+m-1}[y(t)-y(0)]=t^{\mu+m-1}[y(t)-g(0)]
$$

to change (1.1) to the equation

$$
\tilde{y}(t)=\tilde{g}(t)+t^{\mu+m-1} \int_{0}^{t} s^{1-m-\mu}(t-s)^{-\mu} K(t, s) \tilde{y}(s) d s, \quad 0 \leq t \leq T,
$$

where

$$
\tilde{g}(t)=g(0) \cdot t^{\mu+m-1} \int_{0}^{t}(t-s)^{-\mu} K(t, s) d s .
$$

It is easy to see that the solution of (1.5) satisfies

$$
\tilde{y}(t) \in C^{m}([0, T]) \text {. }
$$

To use the theory of orthogonal polynomials, we make the change of variables

$$
t=\frac{T}{2}(1+x), \quad x=\frac{2}{T} t-1,
$$

to rewrite problem (1.5) as follows:

$$
u(x)=f(x)+\int_{0}^{\frac{T}{2}(1+x)} s^{-\mu}\left(\frac{T}{2}(1+x)-s\right)^{-\mu} \widehat{K}(x, s) \tilde{y}(s) d s,
$$

where $x \in[-1,1]$,

$$
u(x)=\tilde{y}(T(1+x) / 2), \quad f(x)=\tilde{g}(T(1+x) / 2),
$$

and

$$
\widehat{K}(x, s)=s^{1-m} \cdot\left[\frac{T}{2}(1+x)\right]^{\mu+m-1} \cdot K\left(\frac{T}{2}(1+x), s\right) .
$$

By using a linear transformation:

$$
s=\frac{T}{2}(1+\tau), \quad \tau \in[-1, x],
$$


the equation (1.9) becomes

$$
u(x)=f(x)+\int_{-1}^{x}(1+\tau)^{-\mu}(x-\tau)^{-\mu} \widetilde{K}(x, \tau) u(\tau) d \tau,
$$

for $x \in[-1,1]$, where

$$
\begin{aligned}
& \widetilde{K}(x, \tau)=(1+\tau)^{1-m}(1+x)^{\mu+m-1} \bar{K}(x, \tau), \\
& \bar{K}(x, \tau)=\left(\frac{T}{2}\right)^{1-\mu} K\left(\frac{T}{2}(1+x), \frac{T}{2}(1+\tau)\right) \in C^{m}([-1,1] \times[-1,1]) .
\end{aligned}
$$

Recently, in 28, a Legendre-collocation method was proposed to solve the Volterra integral equations of the second kind whose kernel and solutions are sufficiently smooth. Then, in [7, a Chebyshev-collocation method was proposed and analyzed for the special case $\mu=\frac{1}{2}$ for (1.1). The main purpose of this work is to use Jacobicollocation methods to numerically solve the Volterra integral equations (1.12). We will provide a rigorous error analysis, which theoretically justifies the spectral rate of convergence of the proposed method.

The paper is organized as follows. In Section 2, we introduce the Jacobicollocation spectral approaches for the Volterra integral equations (1.12). Some preliminaries and useful lemmas are provided in Section 3. The convergence analysis is given in Section 4 . We prove the error estimates in the $L^{\infty}$-norm and the weighted $L^{2}$-norm. The numerical experiments are carried out in Section 5 , which will be used to verify the theoretical results obtained in Section 4 . The final section contains conclusions and remarks.

Throughout the paper, $C$ will denote a generic positive constant that is independent of $N$ but which will depend on $T$ and on the bounds for the given functions $g$ and $K$.

\section{JACOBI-COLLOCATION METHODS}

Let $\omega^{\alpha, \beta}(x)=(1-x)^{\alpha}(1+x)^{\beta}$ be a weight function in the usual sense, for $\alpha, \beta>-1$. As defined in [6, 12, 13, 14, 25, 30, the set of Jacobi polynomials $\left\{J_{n}^{\alpha, \beta}(x)\right\}_{n=0}^{\infty}$ forms a complete $L_{\omega^{\alpha, \beta}}^{2}(-1,1)$-orthogonal system, where $L_{\omega^{\alpha, \beta}}^{2}(-1,1)$ is a weighted space defined by

$$
L_{\omega^{\alpha, \beta}}^{2}(-1,1)=\left\{v: v \text { is measurable and }\|v\|_{\omega^{\alpha, \beta}}<\infty\right\},
$$

equipped with the norm

$$
\|v\|_{\omega^{\alpha, \beta}}=\left(\int_{-1}^{1}|v(x)|^{2} \omega^{\alpha, \beta}(x) d x\right)^{\frac{1}{2}}
$$

and the inner product

$$
(u, v)_{\omega^{\alpha, \beta}}=\int_{-1}^{1} u(x) v(x) \omega^{\alpha, \beta}(x) d x, \quad \forall u, v \in L_{\omega^{\alpha, \beta}}^{2}(-1,1) .
$$

For a given positive integer $N$, we denote the collocation points by $\left\{x_{i}\right\}_{i=0}^{N}$, which is the set of $(N+1)$ Jacobi Gauss, or Jacobi Gauss-Radau, or Jacobi Gauss-Lobatto points, and by $\left\{w_{i}\right\}_{i=0}^{N}$ the corresponding weights. Let $\mathcal{P}_{N}$ denote the space of all polynomials of degree not exceeding $N$. For any $v \in C[-1,1]$, see, e.g., [6, 13, 14, 25], we can define the Lagrange interpolating polynomial $I_{N}^{\alpha, \beta} v \in \mathcal{P}_{N}$, satisfying

$$
I_{N}^{\alpha, \beta} v\left(x_{i}\right)=v\left(x_{i}\right), \quad 0 \leq i \leq N .
$$


It can be written as an expression of the form

$$
I_{N}^{\alpha, \beta} v(x)=\sum_{i=0}^{N} v\left(x_{i}\right) F_{i}(x),
$$

where $F_{i}(x)$ is the Lagrange interpolation basis function associated with the Jacobicollocation points $\left\{x_{i}\right\}_{i=0}^{N}$.

Firstly, assume that the equation (1.12) holds at the collocation points $\left\{x_{i}\right\}_{i=0}^{N}$ on $[-1,1]$, namely,

$$
u\left(x_{i}\right)=f\left(x_{i}\right)+\int_{-1}^{x_{i}}(1+\tau)^{-\mu}\left(x_{i}-\tau\right)^{-\mu} \widetilde{K}\left(x_{i}, \tau\right) u(\tau) d \tau
$$

for $0 \leq i \leq N$. In order to obtain high-order accuracy of the approximated solution, we use the Gauss-type quadrature formula relative to the Jacobi weight with $\alpha=$ $\beta=-\mu$ to compute the integral term in (2.1). Based on this idea, we need to transfer the integral interval $\left[-1, x_{i}\right]$ to the unit interval $[-1,1]$,

$$
\int_{-1}^{x_{i}}(1+\tau)^{-\mu}\left(x_{i}-\tau\right)^{-\mu} \widetilde{K}\left(x_{i}, \tau\right) u(\tau) d \tau=\int_{-1}^{1}\left(1-\theta^{2}\right)^{-\mu} K_{1}\left(x_{i}, \tau_{i}(\theta)\right) u\left(\tau_{i}(\theta)\right) d \theta,
$$

by using the transformation

$$
\tau=\tau_{i}(\theta)=\frac{1+x_{i}}{2} \theta+\frac{x_{i}-1}{2}, \quad \theta \in[-1,1] .
$$

Here,

$$
K_{1}\left(x_{i}, \tau_{i}(\theta)\right)=\left(\frac{1+x_{i}}{2}\right)^{1-2 \mu} \widetilde{K}\left(x_{i}, \tau_{i}(\theta)\right) .
$$

Next, using an $(N+1)$-point Gauss quadrature formula relative to the Jacobi weight $\left\{w_{i}\right\}_{i=0}^{N}$, the integration term in (2.2) can be approximated by

$$
\left.\int_{-1}^{1}\left(1-\theta^{2}\right)^{-\mu} K_{1}\left(x_{i}, \tau_{i}(\theta)\right) u\left(\tau_{i}(\theta)\right)\right) d \theta \sim \sum_{k=0}^{N} K_{1}\left(x_{i}, \tau_{i}\left(\theta_{k}\right)\right) u\left(\tau_{i}\left(\theta_{k}\right)\right) w_{k}
$$

where the set $\left\{\theta_{k}\right\}_{k=0}^{N}$ coincides with the collocation points $\left\{x_{i}\right\}_{i=0}^{N}$ on $[-1,1]$. We use $u_{i}, 0 \leq i \leq N$, to indicate the approximate value for $u\left(x_{i}\right), 0 \leq i \leq N$, and use

$$
u^{N}(x)=\sum_{j=0}^{N} u_{j} F_{j}(x)
$$

to approximate the function $u(x)$, namely,

$$
u\left(x_{i}\right) \sim u_{i}, \quad u(x) \sim u^{N}(x), \quad u\left(\tau_{i}\left(\theta_{k}\right)\right) \sim \sum_{j=0}^{N} u_{j} F_{j}\left(\tau_{i}\left(\theta_{k}\right)\right) .
$$

The Jacobi-collocation method is to seek $u^{N}(x)$ such that $\left\{u_{i}\right\}_{i=0}^{N}$ satisfies the following collocation equations:

$$
u_{i}=f\left(x_{i}\right)+\sum_{j=0}^{N} u_{j}\left(\sum_{k=0}^{N} K_{1}\left(x_{i}, \tau_{i}\left(\theta_{k}\right)\right) F_{j}\left(\tau_{i}\left(\theta_{k}\right)\right) w_{k}\right),
$$

for $0 \leq i \leq N$. We denote the error function by

$$
e(x):=\left(u-u^{N}\right)(x), \quad x \in[-1,1] .
$$


It follows from (1.4) and (1.10) that

$$
y(t)=y(0)+\left[\frac{T}{2}(1+x)\right]^{1-m-\mu} u(x) .
$$

Consequently, the approximate solution to (1.1) is given by

$$
y^{N}(t)=y(0)+\left[\frac{T}{2}(1+x)\right]^{1-m-\mu} u^{N}(x) .
$$

Then the corresponding error functions satisfy

$$
\epsilon(t):=\left(y-y^{N}\right)(t)=\left[\frac{T}{2}(1+x)\right]^{1-m-\mu} e(x)=t^{1-m-\mu} e(x) .
$$

\section{SOME PRELIMINARIES AND USEFUl LEMMAS}

We first introduce some weighted Hilbert spaces. For simplicity, denote $\partial_{x} v(x)=$ $(\partial / \partial x) v(x)$, etc. For a nonnegative integer $m$, define

$$
H_{\omega^{\alpha, \beta}}^{m}(-1,1):=\left\{v: \partial_{x}^{k} v \in L_{\omega^{\alpha, \beta}}^{2}(-1,1), 0 \leq k \leq m\right\},
$$

with the semi-norm and the norm as

$$
|v|_{m, \omega^{\alpha, \beta}}=\left\|\partial_{x}^{m} v\right\|_{\omega^{\alpha, \beta}}, \quad\|v\|_{m, \omega^{\alpha, \beta}}=\left(\sum_{k=0}^{m}\left\|\partial_{x}^{k} v\right\|_{\omega^{\alpha, \beta}}^{2}\right)^{1 / 2}
$$

respectively. Let $\omega(x)=\omega^{-\frac{1}{2},-\frac{1}{2}}(x)$ denote the Chebyshev weight function. In bounding some approximation error of Chebyshev polynomials, only some of the $L^{2}$-norms appearing on the right-hand side of the above norm enter into play. Thus, it is convenient to introduce the semi-norms

$$
|v|_{H_{\omega}^{m ; N}(-1,1)}=\left(\sum_{k=\min (m, N+1)}^{m}\left|\partial_{x}^{k} v\right|_{L_{\omega}^{2}(-1,1)}^{2}\right)^{\frac{1}{2}} .
$$

For bounding some approximation error of Jacobi polynomials, we need the following nonuniformly-weighted Sobolev spaces:

$$
H_{\omega^{\alpha, \beta}, *}^{m}(-1,1):=\left\{v: \partial_{x}^{k} v \in L_{\omega^{\alpha+k, \beta+k}}^{2}(-1,1), 0 \leq k \leq m\right\},
$$

equipped with the inner product and the norm as

$$
(u, v)_{m, \omega^{\alpha, \beta}, *}=\sum_{k=0}^{m}\left(\partial_{x}^{k} u, \partial_{x}^{k} v\right)_{\omega^{\alpha+k, \beta+k}}, \quad\|v\|_{m, \omega^{\alpha, \beta}, *}=\sqrt{(v, v)_{m, \omega^{\alpha, \beta}, *}} .
$$

Furthermore, we introduce the orthogonal projection $\pi_{N, \omega^{\alpha, \beta}}: L_{\omega^{\alpha, \beta}}^{2}(-1,1) \rightarrow \mathcal{P}_{N}$, which is a mapping such that for any $v \in L_{\omega^{\alpha, \beta}}^{2}(-1,1)$,

$$
\left(v-\pi_{N, \omega^{\alpha, \beta}} v, \phi\right)_{\omega^{\alpha, \beta}}=0, \quad \forall \phi \in \mathcal{P}_{N} .
$$

The following result follows from Theorem 1.8.1 in [25] and (3.18) in [13]; also see 12 .

Lemma 3.1. Let $\alpha, \beta>-1$. Then for any function $v \in H_{\omega^{\alpha, \beta}, *}^{m}(-1,1)$ and any nonnegative integer $m$, we have

$$
\left\|\partial_{x}^{k}\left(v-\pi_{N, \omega^{\alpha, \beta}} v\right)\right\|_{\omega^{\alpha+k, \beta+k}} \leq C N^{k-m}\left\|\partial_{x}^{m} v\right\|_{\omega^{\alpha+m, \beta+m}}, \quad 0 \leq k \leq m .
$$


In particular,

$$
\left\|v-\pi_{N, \omega^{\alpha, \beta}} v\right\|_{\omega^{\alpha, \beta}} \leq C N^{-1}\|v\|_{1, \omega^{\alpha+1, \beta+1}} .
$$

Applying Theorem 1.8.4 in [25] and Theorems 4.3, 4.7, 4.10 in [14, we have the following optimal error estimate for the interpolation polynomials based on the Jacobi Gauss points, Jacobi Gauss-Radau points, and Gauss-Lobatto points.

Lemma 3.2. For any function $v$ satisfying $v \in H_{\omega^{\alpha, \beta}, *}^{m}(-1,1)$, with $-1<\alpha, \beta<1$, we have

$$
\left\|v-I_{N}^{\alpha, \beta} v\right\|_{\omega^{\alpha, \beta}} \leq C N^{-m}\left\|\partial_{x}^{m} v\right\|_{\omega^{\alpha+m, \beta+m}},
$$

for the Jacobi Gauss points and Jacobi Gauss-Radau points;

$$
\left\|v-I_{N}^{\alpha, \beta} v\right\|_{\omega^{\alpha, \beta}} \leq C N^{-m}\left\|\partial_{x}^{m} v\right\|_{\omega^{\alpha+m-1, \beta+m-1}},
$$

for the Jacobi Gauss-Lobatto points.

Define a discrete inner product, for any continuous functions $u, v$ on $[-1,1]$, by

$$
(u, v)_{N}=\sum_{j=0}^{N} u\left(x_{j}\right) v\left(x_{j}\right) w_{j} .
$$

By Lemmas 3.1 and 3.2, we can obtain an estimate for the integration error produced by a Gauss-type quadrature formula relative to the Jacobi weight.

Lemma 3.3. If $v \in H_{\omega^{\alpha, \beta}, *}^{m}(-1,1)$ for some $m \geq 1$ and $\phi \in \mathcal{P}_{N}$, then for the Jacobi Gauss and Jacobi Gauss-Radau integration we have

$$
\begin{aligned}
\left|(v, \phi)_{\omega^{\alpha, \beta}}-(v, \phi)_{N}\right| & \leq\left\|v-I_{N}^{\alpha, \beta} v\right\|_{\omega^{\alpha, \beta}}\|\phi\|_{\omega^{\alpha, \beta}} \\
& \leq C N^{-m}\left\|\partial_{x}^{m} v\right\|_{\omega^{\alpha+m, \beta+m}}\|\phi\|_{\omega^{\alpha, \beta}},
\end{aligned}
$$

and for the Jacobi Gauss-Lobatto integration, we have

$$
\begin{aligned}
& \left|(v, \phi)_{\omega^{\alpha, \beta}}-(v, \phi)_{N}\right| \\
\leq & C\left(\left\|v-\pi_{N-1, \omega^{\alpha, \beta}} v\right\|_{\omega^{\alpha, \beta}}+\left\|v-I_{N}^{\alpha, \beta} v\right\|_{\omega^{\alpha, \beta}}\right)\|\phi\|_{\omega^{\alpha, \beta}} \\
\leq & C N^{-m}\left\|\partial_{x}^{m} v\right\|_{\omega^{\alpha+m-1, \beta+m-1}}\|\phi\|_{\omega^{\alpha, \beta}} .
\end{aligned}
$$

We have the following result on the Lebesgue constant for the Lagrange interpolation polynomials associated with the zeros of the Jacobi polynomials; see, e.g., 18 .

Lemma 3.4. Let $\left\{F_{j}(x)\right\}_{j=0}^{N}$ be the $N$-th Lagrange interpolation polynomials associated with the Gauss, or Gauss-Radau, or Gauss-Lobatto points of the Jacobi polynomials. Then

$$
\left\|I_{N}^{\alpha, \beta}\right\|_{\infty}:=\max _{x \in[-1,1]} \sum_{j=0}^{N}\left|F_{j}(x)\right|= \begin{cases}\mathcal{O}(\log N), & -1<\alpha, \beta \leq-\frac{1}{2}, \\ \mathcal{O}\left(N^{\gamma+\frac{1}{2}}\right), & \gamma=\max (\alpha, \beta), \text { otherwise. }\end{cases}
$$

We now introduce some notation. For $r \geq 0$ and $\kappa \in[0,1], C^{r, \kappa}([0, T])$ will denote the space of functions whose $r$-th derivatives are Hölder continuous with exponent $\kappa$, endowed with the usual norm $\|\cdot\|_{r, \kappa}$. When $\kappa=0, C^{r, 0}([0, T])$ denotes the space of functions with $r$ continuous derivatives on $[0, T]$, also denoted by $C^{r}([0, T])$, and with norm $\|\cdot\|_{r}$. 
We will make use of a result of Ragozin [21, 22] (see also [11]), which states that, for each nonnegative integer $r$ and $\kappa \in[0,1]$, there exists a constant $C_{r, \kappa}>0$ such that for any function $v \in C^{r, \kappa}([0, T])$, there exists a polynomial function $\mathcal{T}_{N} v \in \mathcal{P}_{N}$ such that

$$
\left\|v-\mathcal{T}_{N} v\right\|_{\infty} \leq C_{r, \kappa} N^{-(r+\kappa)}\|v\|_{r, \kappa},
$$

where $\|\cdot\|_{\infty}$ is the norm of the space $L^{\infty}([0, T])$, and when the function $v \in C([0, T])$ we also denote $\|v\|_{\infty}=\|v\|_{C([0, T])}$. Actually, as stated in [21, 22], $\mathcal{T}_{N}$ is a linear operator from $C^{r, \kappa}([0, T])$ to $\mathcal{P}_{N}$.

For convenience, we define a linear operator with a weakly singular integral kernel:

$$
(\mathcal{M} v)(t)=t^{\mu+m-1} \int_{0}^{t} s^{1-m-\mu}(t-s)^{-\mu} K(t, s) v(s) d s, \quad t \in[0, T] .
$$

We will need the fact that $\mathcal{M}$ is compact as an operator from $C([0, T])$ to $C^{0, \kappa}([0, T])$ for any $0<\kappa<1-\mu$.

Lemma 3.5. Let $m \geq 2$ and $\kappa \in(0,1-\mu)$ and let $\mathcal{M}$ be defined by (3.10). Then, for any function $v(x) \in C([0, T])$, there exists a positive constant $C$ such that

$$
\|\mathcal{M} v\|_{0, \kappa} \leq C\|v\|_{\infty} .
$$

Proof. We only need to prove that $\mathcal{M}$ is Hölder continuous, namely,

$$
\frac{|\mathcal{M} v(\hat{t})-\mathcal{M} v(\check{t})|}{|\hat{t}-\check{t}|^{\kappa}} \leq C|| v \|_{\infty} \quad 0 \leq \hat{t}<\check{t} \leq T,
$$

for $0<\check{t}-\hat{t}<1$ and $\kappa \in(0,1-\mu)$. Let

$$
k(t, s)=s^{1-m-\mu}(t-s)^{-\mu} K(t, s) .
$$

We then have

$$
\begin{aligned}
& \frac{|\mathcal{M} v(\hat{t})-\mathcal{M} v(\check{t})|}{(\check{t}-\hat{t})^{\kappa}} \\
= & (\check{t}-\hat{t})^{-\kappa}\left|\hat{t}^{\mu+m-1} \int_{0}^{\hat{t}} k(\hat{t}, s) v(s) d s-\check{t}^{\mu+m-1} \int_{0}^{\check{t}} k(\check{t}, s) v(s) d s\right| \\
\leq & E_{1}+E_{2}+E_{3},
\end{aligned}
$$

where

$$
\begin{aligned}
& E_{1}=(\check{t}-\hat{t})^{-\kappa} \hat{t}^{\mu+m-1} \int_{0}^{\hat{t}}|k(\hat{t}, s)-k(\check{t}, s)||v(s)| d s, \\
& E_{2}=(\check{t}-\hat{t})^{-\kappa}\left(\check{t}^{\mu+m-1}-\hat{t}^{\mu+m-1}\right) \int_{0}^{\check{t}}|k(\check{t}, s)||v(s)| d s, \\
& E_{3}=(\check{t}-\hat{t})^{-\kappa} \hat{t}^{\mu+m-1} \int_{\hat{t}}^{\check{t}}|k(\check{t}, s)||v(s)| d s .
\end{aligned}
$$

We now estimate the three terms one by one. Observe

$$
E_{1} \leq E_{1}^{(1)}+E_{1}^{(2)},
$$


where

$$
\begin{aligned}
& E_{1}^{(1)}=(\check{t}-\hat{t})^{-\kappa} \hat{t}^{\mu+m-1} \int_{0}^{\hat{t}} s^{1-m-\mu}\left[(\hat{t}-s)^{-\mu}-(\check{t}-s)^{-\mu}\right]|K(\hat{t}, s)||v(s)| d s, \\
& E_{1}^{(2)}=(\check{t}-\hat{t})^{-\kappa} \hat{t}^{\mu+m-1} \int_{0}^{\hat{t}} s^{1-m-\mu}(\check{t}-s)^{-\mu}|K(\hat{t}, s)-K(\check{t}, s)||v(s)| d s .
\end{aligned}
$$

Recall the definition of the Beta function

$$
\int_{0}^{1} x^{a-1}(1-x)^{b-1} d x=B(a, b), \quad a, b>0 .
$$

This gives that

$$
\int_{0}^{z} \tau^{a-1}(z-\tau)^{b-1} d \tau=z^{a+b-1} B(a, b)
$$

Observe that

$$
\begin{aligned}
\int_{0}^{\hat{t}} s^{1-m-\mu}\left[(\hat{t}-s)^{-\mu}-(\check{t}-s)^{-\mu}\right] d s \\
\quad=B_{1} \hat{t}^{2(1-\mu)-m}-\int_{0}^{\hat{t}} s^{1-m-\mu}(\check{t}-s)^{-\mu} d s \\
\quad=B_{1}\left(\hat{t}^{2(1-\mu)-m}-\check{t}^{2(1-\mu)-m}\right)+\int_{\hat{t}}^{\check{t}} s^{1-m-\mu}(\check{t}-s)^{-\mu} d s,
\end{aligned}
$$

where

$$
B_{1}=B(2-m-\mu, 1-\mu) .
$$

When $m \geq 2, m-2(1-\mu) \geq 0$, we have

$$
\begin{aligned}
& \hat{t}^{\mu+m-1}\left(\hat{t}^{2(1-\mu)-m}-\check{t}^{2(1-\mu)-m}\right) \\
& =\hat{t}^{\mu+m-1} \cdot(m-2(1-\mu)) \xi^{1-2 \mu-m}(\check{t}-\hat{t}) \quad \xi \in(\hat{t}, \check{t}) \\
& =(m-2(1-\mu))(\check{t}-\hat{t}) \cdot\left(\frac{\hat{t}}{\xi}\right)^{\mu+m-1} \cdot \xi^{-\mu} \\
& \leq(m-2(1-\mu))(\check{t}-\hat{t}) \cdot\left(\frac{\hat{t}}{\xi}\right)^{\mu+m-1} \cdot \hat{t}^{-\mu} \\
& \leq(m-2(1-\mu))(\check{t}-\hat{t})^{1-\mu}
\end{aligned}
$$

if $\hat{t} \geq \check{t}-\hat{t}$, and

$$
\hat{t}^{\mu+m-1}\left(\hat{t}^{2(1-\mu)-m}-\check{t}^{2(1-\mu)-m}\right)=\hat{t}^{1-\mu}\left[1-\left(\frac{\hat{t}}{\check{t}}\right)^{m-2(1-\mu)}\right] \leq(\check{t}-\hat{t})^{1-\mu}
$$

if $\hat{t}<\check{t}-\hat{t}$. The above observation, together with (3.16), yields

$$
\begin{aligned}
E_{1}^{(1)} & \leq C\|v\|_{\infty}(\check{t}-\hat{t})^{-\kappa}\left[(\check{t}-\hat{t})^{1-\mu}+\int_{\hat{t}}^{\check{t}}\left(\frac{\hat{t}}{s}\right)^{\mu+m-1}(\check{t}-s)^{-\mu} d s\right] \\
& \leq C\|v\|_{\infty}(\check{t}-\hat{t})^{-\kappa}\left[(\check{t}-\hat{t})^{1-\mu}+\int_{\hat{t}}^{\check{t}}(\check{t}-s)^{-\mu} d s\right] \\
& \leq C\|v\|_{\infty}(\check{t}-\hat{t})^{1-\mu-\kappa} \leq C\|v\|_{\infty},
\end{aligned}
$$


for $\kappa \in(0,1-\mu)$. Furthermore, we have

$$
\begin{aligned}
E_{1}^{(2)} & \leq C\|v\|_{\infty} \hat{t}^{\mu+m-1} \int_{0}^{\hat{t}} s^{1-m-\mu}(\check{t}-s)^{-\mu} \frac{|K(\hat{t}, s)-K(\check{t}, s)|}{(\check{t}-\hat{t})^{\kappa}} d s \\
& \leq C\|v\|_{\infty} \max _{s \in[0, T]}\|K(\cdot, s)\| \|_{0, \kappa} \hat{t}^{\mu+m-1} \int_{0}^{\hat{t}} s^{1-m-\mu}(\check{t}-s)^{-\mu} d s \\
& \leq C\|v\|_{\infty} \hat{t}^{\mu+m-1} \int_{0}^{\check{t}} s^{1-m-\mu}(\check{t}-s)^{-\mu} d s \\
& \leq C\|v\|_{\infty} \hat{t}^{\mu+m-1} \cdot \check{t}^{2(1-\mu)-m} B_{1} \\
& \leq C\|v\|_{\infty}\left(\frac{\hat{t}}{\check{t}}\right)^{\mu+m-1} \cdot \check{t}^{1-\mu} \leq C\|v\|_{\infty},
\end{aligned}
$$

where we have used (3.18) and the fact that $\hat{t}<\check{t}$. Under the following condition:

$$
0<\check{t}-\hat{t}<1,
$$

we have for $\kappa \in(0,1-\mu)$,

$$
\begin{aligned}
E_{2} & \leq C\|v\|_{\infty}(\check{t}-\hat{t})^{-\kappa}\left(\check{t}^{\mu+m-1}-\hat{t}^{\mu+m-1}\right) \int_{0}^{\check{t}} s^{1-m-\mu}(\check{t}-s)^{-\mu} d s \\
& =C\|v\|_{\infty}(\check{t}-\hat{t})^{1-\kappa} \cdot(\mu+m-1) \xi^{\mu+m-2} \cdot \check{t}^{2(1-\mu)-m} B_{1} \quad \xi \in(\hat{t}, \check{t}) \\
& \leq C\|v\|_{\infty}(\check{t}-\hat{t})^{1-\kappa} \cdot\left(\frac{\xi}{\check{t}}\right)^{\mu+m-2} \cdot \check{t}^{-\mu} \\
24) & \leq C\|v\|_{\infty}(\check{t}-\hat{t})^{\mu} \cdot \check{t}^{-\mu} \leq C\|v\|_{\infty},
\end{aligned}
$$

due to $\xi \leq \check{t}, \check{t}-\hat{t}<1,1-\kappa>\mu$, and $\hat{t}<\check{t}$. Finally, we have

$$
\begin{aligned}
E_{3} & \leq C\|v\|_{\infty}(\check{t}-\hat{t})^{-\kappa} \hat{t}^{\mu+m-1} \int_{\hat{t}}^{\check{t}} s^{1-m-\mu}(\check{t}-s)^{-\mu} d s \\
& \leq C\|v\|_{\infty}(\check{t}-\hat{t})^{-\kappa} \int_{\hat{t}}^{\check{t}}\left(\frac{\hat{t}}{s}\right)^{\mu+m-1}(\check{t}-s)^{-\mu} d s \\
& \leq C\|v\|_{\infty}(\check{t}-\hat{t})^{-\kappa} \int_{\hat{t}}^{\breve{t}}(\check{t}-s)^{-\mu} d s \\
& \leq C\|v\|_{\infty}(\check{t}-\hat{t})^{1-\mu-\kappa} \leq C\|v\|_{\infty},
\end{aligned}
$$

where we have used the estimate for $E_{1}^{(1)}$, i.e., (3.22). The desired result (3.11) is established by combining (3.14) with the estimates for $E_{1}, E_{2}$, and $E_{3}$ above.

In our analysis, we shall apply the generalization of Gronwall's Lemma. We call such a function $v=v(t)$ locally integrable on the interval $[0, T]$ if for each $t \in[0, T]$, its Lebesgue integral $\int_{0}^{t} v(s) d s$ is finite. The following result can be found in [31].

Lemma 3.6. Suppose that

$$
v(t) \leq w_{*}(t)+w(t) \int_{0}^{t} \varphi(t, s) v(s) d s, \quad t \in[0, T]
$$


where $\varphi w, \varphi w_{*}$, and $\varphi v$ are locally integrable on the interval $[0, T]$. Here, all the functions are assumed to be nonnegative. Then

$$
v(t) \leq w_{*}(t)+w(t)\left(\exp \int_{0}^{t} \varphi(t, s) w(s) d s\right)\left(\int_{0}^{t} \varphi(t, s) w_{*}(s) d s\right), \quad t \in[0, T] .
$$

From Lemma 3.6, we can directly obtain the following result.

Lemma 3.7. Assume that $v(t)$ is a nonnegative, locally integrable function defined on $([0, T]$ and satisfying

$$
v(t) \leq w_{*}(t)+K_{0} t^{\mu+m-1} \int_{0}^{t} s^{1-m-\mu}(t-s)^{-\mu} v(s) d s, \quad t \in[0, T],
$$

where $K_{0}$ is a positive constant and $w_{*}(t)$ is a nonnegative and continuous function defined on $([0, T]$. Then, there exists a constant $C$ such that

$$
v(t) \leq w_{*}(t)+C t^{\mu+m-1} \int_{0}^{t} s^{1-m-\mu}(t-s)^{-\mu} w_{*}(s) d s, \quad t \in[0, T] .
$$

Proof. Set

$$
w(t)=K_{0} t^{\mu+m-1}, \quad \varphi(t, s)=s^{1-m-\mu}(t-s)^{-\mu} .
$$

It is obvious to see that the conditions in Lemma 3.6 are satisfied in this case. We only need to estimate the term:

$$
\exp \int_{0}^{t} \varphi(t, s) w(s) d s .
$$

Clearly,

$$
\begin{aligned}
\left|\int_{0}^{t} \varphi(t, s) w(s) d s\right| & =\left|K_{0} \int_{0}^{t} s^{1-m-\mu}(t-s)^{-\mu} \cdot s^{\mu+m-1} d s\right| \\
& \leq K_{0} \int_{0}^{t}(t-s)^{-\mu} d s \leq C .
\end{aligned}
$$

Thus, the desired result follows from Lemma 3.6.

To prove the error estimate in the weighted $L^{2}$-norm, we need the generalized Hardy's inequality with weights (see, e.g., [10, 16, 24]).

Lemma 3.8. For all measurable function $f \geq 0$, the following generalized Hardy's inequality

$$
\left(\int_{a}^{b}|(\mathcal{K} f)(x)|^{q} \omega_{1}(x) d x\right)^{1 / q} \leq C\left(\int_{a}^{b}|f(x)|^{p} \omega_{2}(x) d x\right)^{1 / p}
$$

holds if and only if

$$
\sup _{a<x<b}\left(\int_{x}^{b} \omega_{1}(t) d t\right)^{1 / q}\left(\int_{a}^{x} \omega_{2}^{1-p^{\prime}}(t) d t\right)^{1 / p^{\prime}}<\infty, \quad p^{\prime}=\frac{p}{p-1}
$$

for the case $1<p \leq q<\infty$. Here, $\mathcal{K}$ is an operator of the form

$$
(\mathcal{K} f)(x)=\int_{a}^{x} \rho(x, t) f(t) d t
$$

with $\rho(x, t)$ a given kernel, $\omega_{1}, \omega_{2}$ weight functions, and $-\infty \leq a<b \leq \infty$. 
Using Theorem 1 in [19, we have the following estimate for the Lagrange interpolation associated with the Jacobi Gaussian collocation points.

Lemma 3.9. For every bounded function $v(x)$, there exists a constant $C$ independent of $v$ such that

$$
\sup _{N}\left\|\sum_{j=0}^{N} v\left(x_{j}\right) F_{j}(x)\right\|_{L_{\omega^{\alpha}, \beta}^{2}(-1,1)} \leq C\|v\|_{\infty},
$$

where $F_{i}(x)$ is the Lagrange interpolation basis function associated with the Jacobicollocation points $\left\{x_{i}\right\}_{i=0}^{N}$.

\section{Convergence analysis}

The objective of this section is to analyze the approximation scheme (2.6). Firstly, we derive the error estimate in the $L^{\infty}$-norm of the Jacobi-collocation method. Before we state the main results, the following regularity result of the kernel function $K_{1}$ needs to be proved.

Lemma 4.1. Let $\left\{x_{i}\right\}_{i=0}^{N}$ be the set of $(N+1)$ Jacobi Gauss, or Jacobi GaussRadau, or Jacobi Gauss-Lobatto points, for $\alpha=\beta=-\mu$. Then, we have that

$$
\partial_{\theta}^{m} K_{1}\left(x_{i}, \tau_{i}(\theta)\right) \in L_{\omega^{m-\mu, m-\mu}}^{2}(-1,1),
$$

for the Jacobi Gauss points and Jacobi Gauss-Radau points;

$$
\partial_{\theta}^{m} K_{1}\left(x_{i}, \tau_{i}(\theta)\right) \in L_{\omega^{m-\mu-1, m-\mu-1}}^{2}(-1,1),
$$

for the Jacobi Gauss-Lobatto points. Thus, it is reasonable to denote

$$
K^{*}=\max _{0 \leq i \leq N}\left\|\partial_{\theta}^{m} K_{1}\left(x_{i}, \tau_{i}(\cdot)\right)\right\|_{\omega^{m-\mu, m-\mu}},
$$

for the Jacobi Gauss points and Jacobi Gauss-Radau points;

$$
K^{*}=\max _{0 \leq i \leq N}\left\|\partial_{\theta}^{m} K_{1}\left(x_{i}, \tau_{i}(\cdot)\right)\right\|_{\omega^{m-\mu-1, m-\mu-1}},
$$

for the Jacobi Gauss-Lobatto points. Here $\tau_{i}(\theta)$ is given by (2.3) and $K_{1}\left(x_{i}, \tau_{i}(\theta)\right)$ is defined by (2.4), (1.13)-(1.14) for any $0 \leq i \leq N$.

Proof. It follows from (2.4) and (1.13)-(1.14) that

$$
\partial_{\theta}^{m} K_{1}\left(x_{i}, \tau_{i}(\theta)\right)=\left(1+\tau_{i}(\theta)\right)^{1-2 m}\left(1+x_{i}\right)^{2 m-\mu} v\left(x_{i}, \tau_{i}(\theta)\right),
$$

where $v\left(x_{i}, \tau_{i}(\theta)\right)$ is a smooth function with respect to $\theta$ on the interval $[-1,1]$. Now, we use the transformation

$$
\theta=\frac{2}{t_{i}} s-1, \quad s \in\left[0, t_{i}\right]
$$

to derive that

$$
\begin{aligned}
& \left\|\partial_{\theta}^{m} K_{1}\left(x_{i}, \tau_{i}(\cdot)\right)\right\|_{\omega^{m-\mu, m-\mu}}^{2}=\int_{-1}^{1}\left(1-\theta^{2}\right)^{m-\mu}\left|\partial_{\theta}^{m} K_{1}\left(x_{i}, \tau_{i}(\theta)\right)\right|^{2} d \theta \\
& \quad=\int_{0}^{t_{i}} s^{m-\mu}\left(t_{i}-s\right)^{m-\mu}\left(\frac{2}{t_{i}}\right)^{2(m-\mu)+1}\left(\frac{2}{T} s\right)^{2-4 m}\left(\frac{2}{T} t_{i}\right)^{4 m-2 \mu}|v|^{2} d s \\
& \quad \leq C\|v\|_{\infty} t_{i}^{2 m+1} \int_{0}^{t_{i}} s^{2-3 m-\mu}\left(t_{i}-s\right)^{m-\mu} d s \\
& \quad \leq C\|v\|_{\infty} t_{i}^{2 m+1} \cdot t_{i}^{3-2 m-2 \mu}=C\|v\|_{\infty} t_{i}^{4-2 \mu} \leq C
\end{aligned}
$$


for the Jacobi Gauss points and Jacobi Gauss-Radau points, where the Beta function (3.18) was applied. For the Jacobi Gauss-Lobatto points, it is similarly the case that

$$
\left\|\partial_{\theta}^{m} K_{1}\left(x_{i}, \tau_{i}(\cdot)\right)\right\|_{\omega^{m-\mu-1, m-\mu-1}}^{2} \leq C\|v\|_{\infty} t_{i}^{2(1-\mu)} \leq C .
$$

The desired result is now obtained.

Theorem 4.1. Let $u$ be the exact solution of the Volterra equation (1.12). Assume that the approximated solution $u^{N}$ of the form (2.5) is given by the spectralcollocation scheme (2.6) with the Jacobi Gauss, or Jacobi Gauss-Radau, or Jacobi Gauss-Lobbatto collocation points. If the given data $g(t)$ and $K(t, s)$ in (1.1) satisfy $g(t) \in C^{m}([0, T])$ and $K(t, s) \in C^{m}([0, T] \times[0, T])(m \geq 2)$, then for sufficiently large $N$,

$$
\left\|u-u^{N}\right\|_{\infty} \leq \begin{cases}C N^{\frac{1}{2}-m} \log N\left(|u|_{H_{\omega}^{m ; N}(-1,1)}+N^{-\frac{1}{2}} K^{*}\|u\|_{\infty}\right), & \frac{1}{2}<\mu<1, \\ C N^{\frac{1}{2}-m}\left(|u|_{H_{\omega}^{m ; N}(-1,1)}+N^{-\frac{1}{2}} \log N K^{*}\|u\|_{\infty}\right), & \mu=\frac{1}{2}, \\ C N^{1-\mu-m}\left(|u|_{H_{\omega}^{m ; N}(-1,1)}+N^{-\frac{1}{2}} K^{*}|| u \|_{\infty}\right), & 0<\mu<\frac{1}{2},\end{cases}
$$

where $K^{*}$ is defined by (4.1)-(4.2).

Proof. Since the given data $g(t)$ and $K(t, s)$ in (1.1) satisfy $g(t) \in C^{m}([0, T])$ and $K(t, s) \in C^{m}([0, T] \times[0, T])(m \geq 2)$, we have $u \in C^{m}([-1,1])$ based on the analysis in Section 1. Consequently, $u \in H_{\omega}^{m}(-1,1) \cap L^{\infty}(-1,1)$. By using (2.1)-(2.2) and the definition of the weighted inner product, we first observe that the solution $u$ of (1.12) satisfies

$$
u\left(x_{i}\right)=f\left(x_{i}\right)+\left(K_{1}\left(x_{i}, \tau_{i}(\cdot)\right), u\left(\tau_{i}(\cdot)\right)\right)_{\omega^{-\mu,-\mu}}
$$

for $0 \leq i \leq N$. By using the definition of the discrete inner product (3.3), we set

$$
\left(K_{1}\left(x_{i}, \tau_{i}(\cdot)\right), \phi\left(\tau_{i}(\cdot)\right)\right)_{N}=\sum_{k=0}^{N} K_{1}\left(x_{i}, \tau_{i}\left(\theta_{k}\right)\right) \phi\left(\tau_{i}\left(\theta_{k}\right)\right) w_{k} .
$$

Then, the numerical scheme (2.6) can be written as

$$
u_{i}=f\left(x_{i}\right)+\left(K_{1}\left(x_{i}, \tau_{i}(\cdot)\right), u^{N}\left(\tau_{i}(\cdot)\right)\right)_{N},
$$

for $0 \leq i \leq N$, where $u^{N}$ is defined by (2.5). We now subtract (4.8) from (4.7) to get the error equation

$$
\begin{aligned}
u\left(x_{i}\right)-u_{i} & =\left(K_{1}\left(x_{i}, \tau_{i}(\cdot)\right), e\left(\tau_{i}(\cdot)\right)\right)_{\omega^{-\mu,-\mu}}+I_{i, 2} \\
& =\int_{-1}^{x_{i}}(1+\tau)^{-\mu}\left(x_{i}-\tau\right)^{-\mu} \widetilde{K}\left(x_{i}, \tau\right) e(\tau) d \tau+I_{i, 2},
\end{aligned}
$$

for $0 \leq i \leq N, e(x)=u(x)-u^{N}(x)$ is the error function, and

$$
I_{i, 2}=\left(K_{1}\left(x_{i}, \tau_{i}(\cdot)\right), u^{N}\left(\tau_{i}(\cdot)\right)\right)_{\omega^{-\mu,-\mu}}-\left(K_{1}\left(x_{i}, \tau_{i}(\cdot)\right), u^{N}\left(\tau_{i}(\cdot)\right)\right)_{N} .
$$

In (4.9), the integral transformation (2.2) was used. Applying again the transformation (1.8) and (1.11), we change (4.9) to

$$
u\left(x_{i}\right)-u_{i}=t_{i}^{\mu+m-1} \int_{0}^{t_{i}} s^{1-m-\mu}\left(t_{i}-s\right)^{-\mu} K\left(t_{i}, s\right) \tilde{e}(s) d s+I_{i, 2},
$$


where

$$
\begin{gathered}
\tilde{e}(t)=e\left(\frac{2}{T} t-1\right), \\
t_{i}=\frac{T}{2}\left(1+x_{i}\right), \quad \text { for } 0 \leq i \leq N .
\end{gathered}
$$

Multiplying $F_{j}(x)$ on both sides of the error equation (4.10) and summing up from $i=0$ to $i=N$ yield

$$
I_{N}^{-\mu,-\mu} u-u^{N}=\sum_{i=0}^{N}(\mathcal{M} \tilde{e})\left(t_{i}\right) F_{i}(x)+\sum_{i=0}^{N} I_{i, 2} F_{i}(x),
$$

where $\mathcal{M}$ was defined in (3.10). Consequently, recalling the relation of the error function (2.10) gives that

$$
\tilde{e}(t)=t^{\mu+m-1} \int_{0}^{t} s^{1-m-\mu}(t-s)^{-\mu} K(t, s) \tilde{e}(s) d s+I_{1}+I_{2}+I_{3},
$$

where

$$
I_{1}=u-I_{N}^{-\mu,-\mu} u, \quad I_{2}=\sum_{i=0}^{N} I_{i, 2} F_{i}(x), \quad I_{3}=I_{N}^{-\mu,-\mu}(\mathcal{M} \tilde{e})-\mathcal{M} \tilde{e} .
$$

From (4.12), we have

$$
|\tilde{e}(t)| \leq w_{*}(t)+K_{0} t^{\mu+m-1} \int_{0}^{t} s^{1-m-\mu}(t-s)^{-\mu}|\tilde{e}(s)| d s, \quad t \in[0, T],
$$

where

$$
w_{*}(t)=\left|I_{1}+I_{2}+I_{3}\right|, \quad K_{0}=\max _{0 \leq s<t \leq T}|K(t, s)|
$$

Using the generalized Gronwall inequality, i.e., Lemma 3.7. we have

$$
|\tilde{e}(t)| \leq w_{*}(t)+C t^{\mu+m-1} \int_{0}^{t} s^{1-m-\mu}(t-s)^{-\mu} w_{*}(s) d s, \quad t \in[0, T] .
$$

Then, it follows from (4.15) and (3.18) that

$$
\|e\|_{\infty}=\|\tilde{e}\|_{\infty} \leq C\left\|w_{*}\right\|_{\infty} \leq C\left(\left\|I_{1}\right\|_{\infty}+\left\|I_{2}\right\|_{\infty}+\left\|I_{3}\right\|_{\infty}\right) .
$$

Firstly, let $I_{N}^{c} u \in \mathcal{P}_{N}$ denote the interpolant of $u$ at any of the three families of Chebyshev Gauss points. From (5.5.28) in [6], the interpolation error estimate in the maximum norm is given by

$$
\left\|I_{1}\right\|_{\infty}=\left\|u-I_{N}^{c} u\right\|_{\infty} \leq C N^{1 / 2-m}|u|_{H_{\omega}^{m ; N}(-1,1)}, \quad \text { when } \mu=\frac{1}{2} .
$$

Note that

$$
I_{N}^{-\mu,-\mu} p(x)=p(x), \quad \text { i.e., } \quad\left(I_{N}^{-\mu,-\mu}-I\right) p(x)=0, \quad \forall p(x) \in \mathcal{P}_{N},
$$


where $I$ denotes the identity operator. By using (4.18), Lemma 3.4, and (4.17), we obtain that

$$
\begin{aligned}
\left\|I_{1}\right\|_{\infty} & =\left\|u-I_{N}^{-\mu,-\mu} u\right\|_{\infty} \\
& =\left\|u-I_{N}^{c} u+I_{N}^{-\mu,-\mu}\left(I_{N}^{c} u\right)-I_{N}^{-\mu,-\mu} u\right\|_{\infty} \\
& \leq\left\|u-I_{N}^{c} u\right\|_{\infty}+\left\|I_{N}^{-\mu,-\mu}\left(I_{N}^{c} u-u\right)\right\|_{\infty} \\
& \leq\left(1+\left\|I_{N}^{-\mu,-\mu}\right\|_{\infty}\right)\left\|u-I_{N}^{c} u\right\|_{\infty} \\
& \leq \begin{cases}C N^{\frac{1}{2}-m} \log N|u|_{H_{\omega}^{m ; N}(-1,1)}, & \frac{1}{2}<\mu<1, \\
C N^{1-\mu-m}|u|_{H_{\omega}^{m ; N}(-1,1)}, & 0<\mu<\frac{1}{2} .\end{cases}
\end{aligned}
$$

Next, it follows from Lemma 3.3 that

$$
\left|I_{i, 2}\right| \leq C N^{-m}|| \partial_{\theta}^{m} K_{1}\left(x_{i}, \tau_{i}(\cdot)\right)\left\|_{\omega^{m-\mu-l, m-\mu-l}}\right\| u^{N}\left(\tau_{i}(\cdot)\right) \|_{\omega^{-\mu,-\mu}},
$$

so that

$$
\begin{aligned}
& \max _{0 \leq i \leq N}\left|I_{i, 2}\right| \\
& \leq C N^{-m} \max _{0 \leq i \leq N}\left\|\partial_{\theta}^{m} K_{1}\left(x_{i}, \tau_{i}(\cdot)\right)\right\|_{\omega^{m-\mu-l, m-\mu-l}} \max _{0 \leq i \leq N}\left\|u^{N}\left(\tau_{i}(\cdot)\right)\right\|_{\omega^{-\mu,-\mu}} \\
& \leq C N^{-m} K^{*}\left\|u^{N}\right\|_{\infty} \leq C N^{-m} K^{*}\left(\|e\|_{\infty}+\|u\|_{\infty}\right),
\end{aligned}
$$

where $K^{*}$ is defined by (4.1)- (4.2), $l=0$ for the Jacobi Gauss points and Jacobi Gauss-Radau points, and $l=1$ for the Jacobi Gauss-Lobatto points. Hence, by using Lemma 3.4 and (4.21), we have

$$
\begin{gathered}
\left\|I_{2}\right\|_{\infty}=\left\|\sum_{i=0}^{N} I_{i, 2} F_{i}(x)\right\|_{\infty} \leq C \max _{0 \leq i \leq N}\left|I_{i, 2}\right| \max _{x \in[-1,1]} \sum_{j=0}^{N}\left|F_{j}(x)\right| \\
\leq \begin{cases}C N^{-m} \log N K^{*}\left(\|e\|_{\infty}+\|u\|_{\infty}\right), & \frac{1}{2} \leq \mu<1, \\
C N^{\frac{1}{2}-\mu-m} K^{*}\left(\|e\|_{\infty}+\|u\|_{\infty}\right), & 0<\mu<\frac{1}{2},\end{cases}
\end{gathered}
$$

for sufficiently large $N$. We now estimate the third term $I_{3}$. It is clear that $\tilde{e} \in$ $C[0, T]$. Consequently, using (3.9) and Lemma 3.5 it follows that

$$
\left\|\mathcal{M} \tilde{e}-\mathcal{T}_{N} \mathcal{M} \tilde{e}\right\|_{\infty} \leq C N^{-\kappa}\|\mathcal{M} \tilde{e}\|_{0, \kappa} \leq C N^{-\kappa}\|\tilde{e}\|_{\infty}=C N^{-\kappa}\|e\|_{\infty}
$$

where $\kappa \in(0,1-\mu)$ and $\mathcal{T}_{N} \mathcal{M} \tilde{e} \in \mathcal{P}_{N}$. It follows from (4.18), Lemma 3.4, and the above estimate that

$$
\begin{aligned}
\left\|I_{3}\right\|_{\infty} & =\left\|\left(I_{N}^{-\mu,-\mu}-I\right) \mathcal{M} \tilde{e}\right\|_{\infty} \\
& =\left\|\left(I_{N}^{-\mu,-\mu}-I\right)\left(\mathcal{M} \tilde{e}-\mathcal{T}_{N} \mathcal{M} \tilde{e}\right)\right\|_{\infty} \\
& \leq\left(1+\left\|I_{N}^{-\mu,-\mu}\right\|_{\infty}\right)\left\|\mathcal{M} \tilde{e}-\mathcal{T}_{N} \mathcal{M} \tilde{e}\right\|_{\infty} \\
& \leq \begin{cases}C N^{-\kappa} \log N\|e\|_{\infty}, \quad \frac{1}{2} \leq \mu<1, \\
C N^{\frac{1}{2}-\kappa-\mu}\|e\|_{\infty}, \quad 0<\mu<\frac{1}{2} .\end{cases}
\end{aligned}
$$

The desired estimate (4.6) follows from (4.16)-(4.17), (4.19), (4.22), and (4.24).

Our next goal is to derive the error estimate in the weighted $L^{2}$-norm. 
Theorem 4.2. Let $u$ and $u^{N}$ be the same as those in Theorem 4.1. If the given data $g(t)$ and $K(t, s)$ in (1.1) satisfy $g(t) \in C^{m}([0, T])$ and $K(t, s) \in C^{m}([0, T] \times[0, T])$ $(m \geq 2)$, then

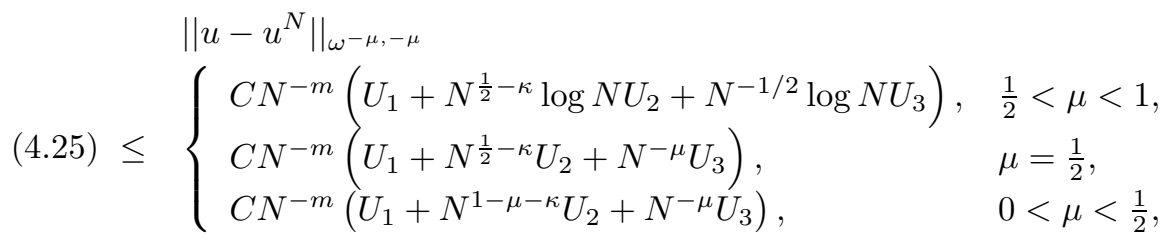

for sufficiently large $N$ and for any $\kappa \in(0,1-\mu)$, where

$$
\begin{aligned}
& U_{1}=\left\|\partial_{x}^{m} u\right\|_{\omega^{m-\mu-l, m-\mu-l}}+\left.K^{*}|| u\right|_{\infty}, \\
& U_{2}=|u|_{H_{\omega}^{m ; N}(-1,1)}, \quad U_{3}=K^{*}|u|_{H_{\omega}^{1}(-1,1)},
\end{aligned}
$$

and $K^{*}$ is defined by (4.1)-(4.2), $l=0$ for the Jacobi Gauss points and Jacobi Gauss-Radau points, and $l=1$ for the Jacobi Gauss-Lobatto points.

Proof. Using the transformation (1.8) and (1.11), we change (4.15) to

$$
|e(x)| \leq w_{*}+C(1+x)^{\mu+m-1} \int_{-1}^{x}(1+\tau)^{1-m-\mu}(x-\tau)^{-\mu} w_{*} d \tau, \quad x \in[-1,1] .
$$

where $w_{*}$ are defined by (4.14). It follows from the generalized Hardy's inequality (Lemma 3.8) that

$$
\|e\|_{\omega^{-\mu,-\mu}} \leq C\left(\left\|I_{1}\right\|_{\omega^{-\mu,-\mu}}+\left\|I_{2}\right\|_{\omega^{-\mu,-\mu}}+\left\|I_{3}\right\|_{\omega^{-\mu,-\mu}}\right) .
$$

Firstly, by Lemma 3.2 we see that

$$
\left\|I_{1}\right\|_{\omega^{-\mu,-\mu}}=\left\|u-I_{N}^{-\mu,-\mu} u\right\|_{\omega^{-\mu,-\mu}} \leq C N^{-m}\left\|\partial_{x}^{m} u\right\|_{\omega^{m-\mu-l, m-\mu-l}} .
$$

Next, it follows from Lemma 3.9 and (4.21) that

$$
\left\|I_{2}\right\|_{\omega^{-\mu,-\mu}}=\left\|\sum_{i=0}^{N} I_{i, 2} F_{i}(x)\right\|_{\omega^{-\mu,-\mu}} \leq C \max _{0 \leq i \leq N}\left|I_{i, 2}\right| \leq C N^{-m} K^{*}\left\|u^{N}\right\|_{\infty} .
$$

By the convergence result in Theorem 4.1, we have

$$
\begin{aligned}
\left\|u^{N}\right\|_{\infty} & \leq\|e\|_{\infty}+\|u\|_{\infty} \\
& \leq \begin{cases}C\left(N^{-1 / 2} \log N|u|_{H_{\omega}^{1}(-1,1)}+\|u\|_{\infty}\right), & \frac{1}{2}<\mu<1, \\
C\left(N^{-\mu}|u|_{H_{\omega}^{1}(-1,1)}+\|u\|_{\infty}\right), & 0<\mu \leq \frac{1}{2}\end{cases}
\end{aligned}
$$

which, together with (4.31), gives

$$
\left\|I_{2}\right\|_{\omega^{-\mu,-\mu}} \leq \begin{cases}C N^{-m} K^{*}\left(N^{-1 / 2} \log N|u|_{H_{\omega}^{1}(-1,1)}+\|u\|_{\infty}\right), & \frac{1}{2}<\mu<1, \\ C N^{-m} K^{*}\left(N^{-\mu}|u|_{H_{\omega}^{1}(-1,1)}+\|u\|_{\infty}\right), & 0<\mu \leq \frac{1}{2},\end{cases}
$$

for sufficiently large $N$. Moreover, it follows from (4.18), Lemma 3.9) and (3.9) that

$$
\begin{aligned}
\left\|I_{3}\right\|_{\omega-\mu,-\mu} & =\left\|\left(I_{N}^{-\mu,-\mu}-I\right) \mathcal{M} \tilde{e}\right\|_{\omega^{-\mu,-\mu}} \\
& =\left\|\left(I_{N}^{-\mu,-\mu}-I\right)\left(\mathcal{M} \tilde{e}-\mathcal{T}_{N} \mathcal{M} \tilde{e}\right)\right\|_{\omega^{-\mu,-\mu}} \\
& \leq\left\|I_{N}^{-\mu,-\mu}\left(\mathcal{M} \tilde{e}-\mathcal{T}_{N} \mathcal{M} \tilde{e}\right)\right\|_{\omega^{-\mu,-\mu}}+\left\|\left(\mathcal{M} \tilde{e}-\mathcal{T}_{N} \mathcal{M} \tilde{e}\right)\right\|_{\omega^{-\mu,-\mu}} \\
& \leq C\left\|\mathcal{M} \tilde{e}-\mathcal{T}_{N} \mathcal{M} \tilde{e}\right\|_{\infty} \leq C N^{-\kappa}\|\mathcal{M} \tilde{e}\|_{0, \kappa} \\
& \leq C N^{-\kappa}\|\tilde{e}\|_{\infty}=C N^{-\kappa}\|e\|_{\infty},
\end{aligned}
$$


where, in the last step we used Lemma 3.5 for any $\kappa \in(0,1-\mu)$. By the convergence result in Theorem 4.1, we obtain that

$$
\left\|I_{3}\right\|_{\omega-\mu,-\mu} \leq \begin{cases}C N^{\frac{1}{2}-\kappa-m} \log N\left(|u|_{H_{\omega}^{m ; N}(-1,1)}+\left.N^{-\frac{1}{2}} K^{*}|| u\right|_{\infty}\right), & \frac{1}{2}<\mu<1, \\ C N^{\frac{1}{2}-\kappa-m}\left(|u|_{H_{\omega}^{m ; N}(-1,1)}+\left.N^{-\frac{1}{2}} \log N K^{*}|| u\right|_{\infty}\right), & \mu=\frac{1}{2}, \\ C N^{1-\mu-\kappa-m}\left(|u|_{H_{\omega}^{m ; N}(-1,1)}+N^{-\frac{1}{2}} K^{*}|| u \|_{\infty}\right), & 0<\mu<\frac{1}{2},\end{cases}
$$

for $N$ sufficiently large and for any $\kappa \in(0,1-\mu)$. The desired estimate (4.25) is obtained by combining (4.29)-(4.30), (4.33) and (4.35).

\section{Numerical EXPERIMENTS}

Let $U_{N}=\left[u_{0}, \ldots, u_{N}\right]^{T}$ and $F_{N}=\left[f\left(x_{0}\right), \ldots, f\left(x_{N}\right)\right]^{T}$. The numerical scheme (2.6) leads to a system of equations of the form

$$
U_{N}=F_{N}+A U_{N},
$$

where the entries of the matrix $A$ are given by

$$
\left.a_{i j}=\left[\frac{T}{2}\left(1+x_{i}\right)\right]^{\mu} \sum_{k=0}^{N} K_{1}\left(x_{i}, \tau_{i}\left(\theta_{k}\right)\right) F_{j}\left(\tau_{i}\left(\theta_{k}\right)\right)\right) w_{k} .
$$

Here, we simply introduce the computation of Gauss-Jacobi quadrature rule nodes and weights (see the detailed algorithm and download related codes in 11). The Gauss-Jacobi quadrature formula is used to numerically calculate the integral

$$
\int_{-1}^{1}(1-x)^{-\mu}(1+x)^{-\mu} f(x) d x, \quad f(x) \in[-1,1],
$$

by using the formula

$$
\int_{-1}^{1}(1-x)^{-\mu}(1+x)^{-\mu} f(x) d x \sim \sum_{i=0}^{N} w_{i} f\left(x_{i}\right) .
$$

With the help of a change in the variables (which changes both weights $w_{i}$ and nodes $x_{i}$ ), we can get onto the arbitrary interval $[a, b]$. If you perform a change of variables, you should take into account that the formula of the error term is changed along with the nodes and weights (you can get a new form by changing the variables in the formula).

The Gauss-Jacobi quadrature formula for a given order $N$ is completely defined by the set of nodes $x_{i}$ and weights $w_{i}$ (see more details in [13, 14]).

TABLE 1. Example 5.1; The $L^{\infty}$ and $L_{\omega}^{2}$ errors for $\tilde{y}(t)$.

\begin{tabular}{||c|c|c|c|c|c||}
\hline $\mathrm{N}$ & 8 & 10 & 12 & 14 & 16 \\
$L^{\infty}$ Error & $9.1871 \mathrm{e}-002$ & $9.6882 \mathrm{e}-003$ & $6.6860 \mathrm{e}-004$ & $3.4915 \mathrm{e}-005$ & $1.3721 \mathrm{e}-006$ \\
$L_{\omega}^{2}$ Error & $2.4140 \mathrm{e}-002$ & $2.9328 \mathrm{e}-003$ & $2.2878 \mathrm{e}-004$ & $1.2547 \mathrm{e}-005$ & $5.1289 \mathrm{e}-007$ \\
\hline $\mathrm{N}$ & 18 & 20 & 22 & 24 & 26 \\
$L^{\infty}$ Error & $4.1937 \mathrm{e}-008$ & $1.0351 \mathrm{e}-009$ & $2.0807 \mathrm{e}-011$ & $5.2591 \mathrm{e}-013$ & $2.1072 \mathrm{e}-013$ \\
$L_{\omega}^{2}$ Error & $1.6280 \mathrm{e}-008$ & $4.1367 \mathrm{e}-010$ & $8.6156 \mathrm{e}-012$ & $2.0914 \mathrm{e}-013$ & $1.3405 \mathrm{e}-013$ \\
\hline
\end{tabular}




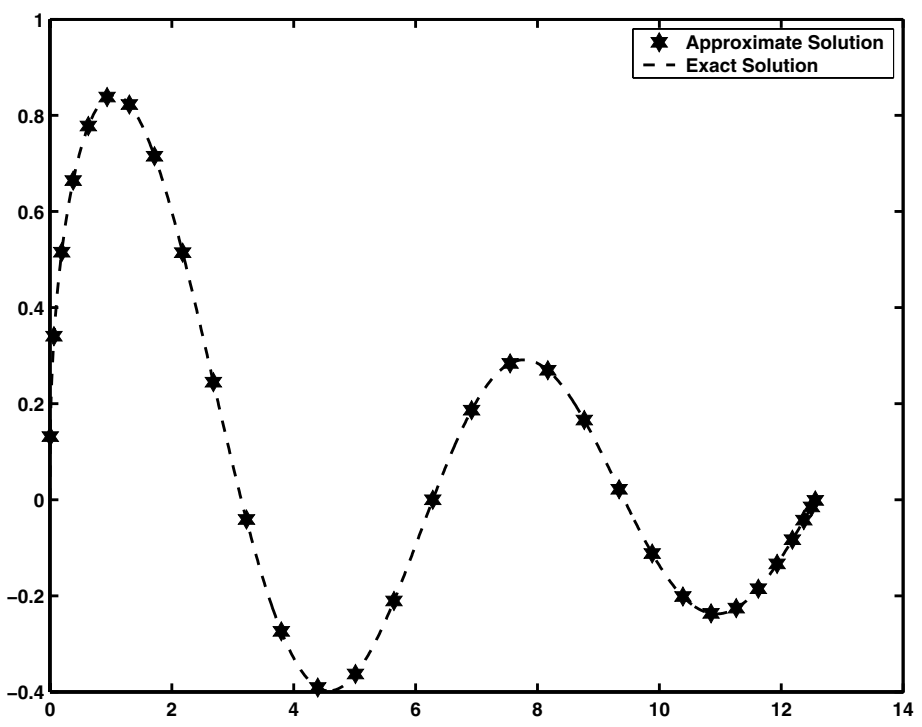

Figure 1. Example 5.1. Numerical and exact solution $y(t)=$ $t^{-m u} \sin (t)$ with $T=4 \pi$ and $\mu=0.6$.

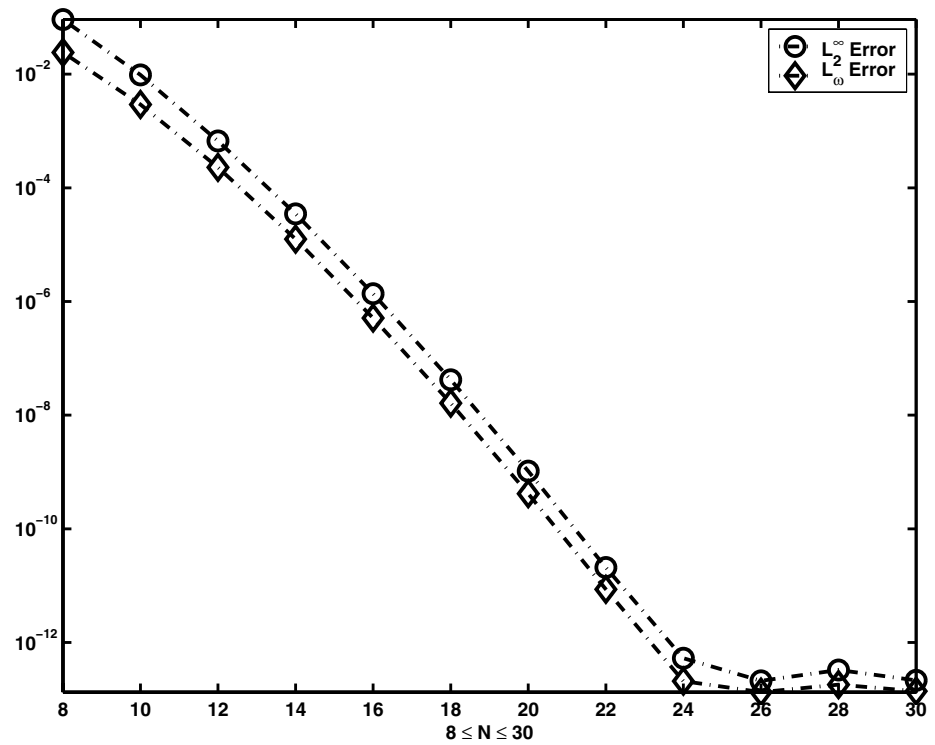

Figure 2. Example 5.1; $L^{\infty}$ and $L_{\omega}^{2}$ errors versus the number of collocation points with the relationship between $t$ and $x, t=$ $\frac{T}{2}(x+1)$. 
Example 5.1. Consider the linear Volterra integral equations of the second kind with a weakly singular kernel:

$$
y(t)=b(t)-\int_{0}^{t}(t-s)^{-\mu} y(s) d s, \quad 0 \leq t \leq T,
$$

with $b(t)$ chosen so that $y(t)=t^{-\mu} \sin t$ for $0<\mu<1$. By calculation,

$$
b(t)=\frac{\sin t}{t^{\mu}}+\sqrt{\pi} \Gamma(1-\mu) t^{1 / 2-\mu} \sin \frac{t}{2} B\left(\frac{1}{2}-\mu, \frac{t}{2}\right),
$$

where $B(\nu, z)$ is the Bessel function defined by

$$
B(\nu, z)=\left(\frac{z}{2}\right)^{\nu} \sum_{k=0}^{\infty} \frac{\left(-\frac{z^{2}}{4}\right)^{k}}{k ! \Gamma(\nu+k+1)} .
$$

This problem has the property stated at the beginning of this paper, i.e., $y^{\prime}(t)=$ $t^{-\mu} \cos t+t^{-\mu-1} \sin t \sim t^{-\mu}$ at $t=0^{+}$, which is singular at $t=0+$. In the theory presented in the previous section, our main concern is the regularity of the transformed solution. For the present problem, if we multiply the solution by the factor $t^{\mu}$, then the resulting function $\tilde{y}(t)=t^{\mu} y(t)=\sin t$ is sufficiently smooth.

Table 1 shows the errors for $\tilde{y}(t)$ obtained by using the spectral methods described above. It is observed that the desired exponential rate of convergence is obtained. Figure 1 presents the numerical and exact solutions for $y$, which are found to be in excellent agreement.

TABle 2. Example 5.2, The $L^{\infty}$ and $L_{\omega}^{2}$ errors for $\tilde{y}(t)$.

\begin{tabular}{||c|c|c|c|c|c||}
\hline $\mathrm{N}$ & 12 & 18 & 24 & 30 & 36 \\
$L^{\infty}$ Error & $7.2640 \mathrm{e}-003$ & $1.4138 \mathrm{e}-003$ & $3.3384 \mathrm{e}-004$ & $5.9624 \mathrm{e}-005$ & $9.1527 \mathrm{e}-006$ \\
$L_{\omega}^{2}$ Error & $9.1299 \mathrm{e}-004$ & $2.2357 \mathrm{e}-004$ & $4.5114 \mathrm{e}-005$ & $7.1458 \mathrm{e}-006$ & $9.9153 \mathrm{e}-007$ \\
\hline $\mathrm{N}$ & 42 & 48 & 54 & 60 & 66 \\
$L^{\infty}$ Error & $1.2507 \mathrm{e}-006$ & $1.5213 \mathrm{e}-007$ & $1.5904 \mathrm{e}-008$ & $1.2551 \mathrm{e}-009$ & $1.4582 \mathrm{e}-010$ \\
$L_{\omega}^{2}$ Error & $1.2435 \mathrm{e}-007$ & $1.4073 \mathrm{e}-008$ & $1.3903 \mathrm{e}-009$ & $1.0726 \mathrm{e}-010$ & $1.2492 \mathrm{e}-011$ \\
\hline
\end{tabular}

Example 5.2. Consider the following nonlinear Volterra integral equation of the second kind with weakly singular kernels:

$$
y(t)=g(t)-\int_{0}^{t}(t-s)^{-\mu} \tan (y(s)) d s, \quad 0 \leq t \leq T,
$$

where

$$
g(t)=\arctan \left(t^{5-\mu}\right)+t^{6-2 \mu} B(11-\mu, 5-\mu) .
$$

There are two issues relevant to the above problem. First, it is a nonlinear Volterra equation. Although the theoretical analysis in this work deals with the linear case only, the method can be extended to handle the nonlinear case quite easily. The implementation follows recent work of Tang and $\mathrm{Xu}$ 29, which uses a Gauss-Seidel-type iteration technique. The second issue is about the regularity. It can be verified that this problem has a unique solution $y(t)=\arctan \left(t^{5-\mu}\right)$. With simple expansions, it is known that

$$
y(t) \sim t^{5-\mu}-\frac{1}{3} t^{3(5-\mu)} .
$$




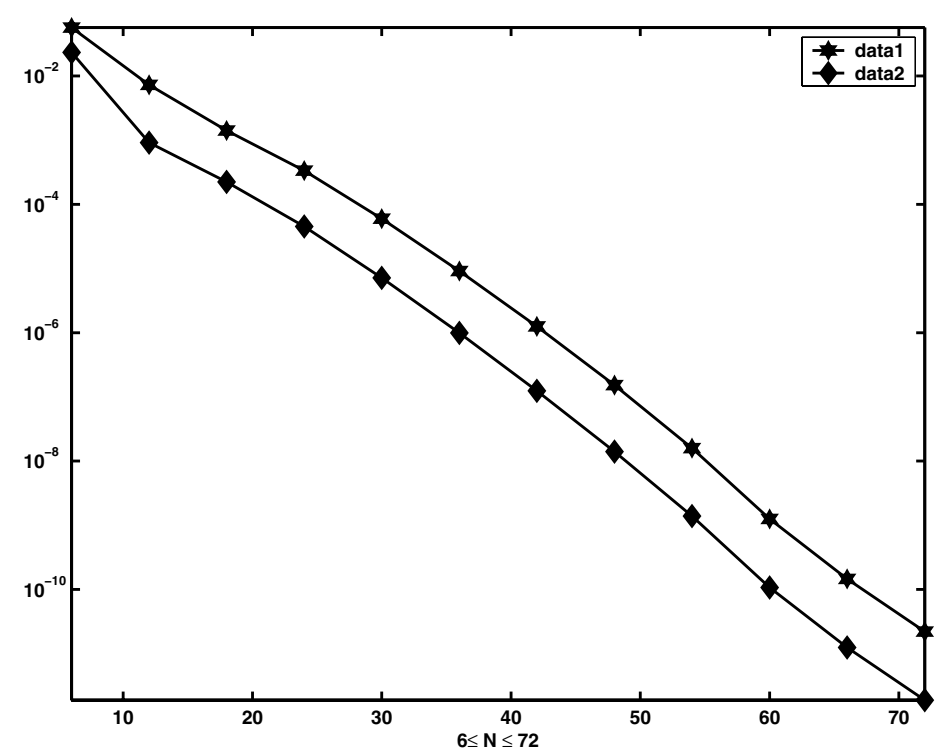

FiguRE 3. Example 5.2, $L^{\infty}$ and $L_{\omega}^{2}$ errors versus the number of collocation points with the relationship between $t$ and $x, t=$ $\frac{T}{2}(x+1)$.

Consequently, a transformation of $t^{\mu+m-1} y(t)$ should be used as suggested by the theoretical analysis in this work. However, the transformation $\tilde{y}(t):=t^{\mu} y(t)$ leads to a smooth function $\tilde{y}(t) \in C^{13}([0, T])$, and this regularity is sufficient in obtaining spectral accuracy with the double-precision machine epsilon.

In Table 2 we present the errors of $\tilde{y}$ for the numerical approximations obtained by using the spectral methods, and Figure 3 plots the corresponding errors for the solutions $y$ of the original equation (5.3).

\section{Conclusion And Future WORK}

This work has been concerned with the Jacobi-collocation spectral analysis of the second kind Volterra integral equations which have a weakly singular kernel of the form $(t-s)^{-\mu}$, where $\mu \in(0,1)$. The derivative $y^{\prime}(t)$ of the solution behaves like $t^{-\mu}$ near the origin, and this is expected to cause a loss in the global convergence order of collocation methods. To this end, the original equation was changed into a new Volterra integral equation which possesses better regularity, by applying some function transformations and variable transformations. Next, we directly presented a discretization scheme for the new Volterra integral equation. We proved the convergence of the method and obtained the error estimates in the $L^{\infty}$-norm and the weighted $L^{2}$-norm of the approximated solution. These results were confirmed by some numerical examples. We have also implemented the Jacobi-collocation method based on the Gauss-Jacobi quadrature formula.

In our future work, the stability will be established for these spectral-collocation methods, and spectral-Galerkin methods also will be studied for Volterra integral equations of the second kind, with a weakly singular kernel. 


\section{ACKNOWLEDGMENT}

The authors are grateful to Mr. Xiang Xu of Fudan University for his assistance in providing us the numerical results in Section 5, and they also thank the referees for their helpful suggestions and comments.

\section{REFERENCES}

[1] S. Bochkanov And V. Bystritsky, Computation of Gauss-Jacobi quadrature rule nodes and weights, http://www.alglib.net/integral/gq/gjacobi.php

[2] H. BRunNer, Nonpolynomial spline collocation for Volterra equations with weakly singular kernels, SIAM J . Numer. Anal., 20 (1983), pp. 1106-1119. MR723827 (85d:65069)

[3] H. Brunner, The numerical solutions of weakly singular Volterra integral equations by collocation on graded meshes, Math. Comp., 45 (1985), pp. 417-437. MR804933 (87b:65223)

[4] H. Brunner, Polynomial spline collocation methods for Volterra integro-differential equations with weakly singular kernels, IMA J . Numer. Anal., 6 (1986), pp. 221-239. MR967664 (89h:65217)

[5] H. Brunner, Collocation Methods for Volterra Integral and Related Functional Differential Equations, Cambridge University Press, 2004. MR2128285(2005k:65002)

[6] C. Canuto, M. Y. Hussaini, A. Quarteroni and T. A. Zang, Spectral Methods. Fundamentals in Single Domains, Springer-Verlag, Berlin, 2006. MR2223552 (2007c:65001)

[7] Y. Chen AND T. TANG, Convergence analysis for the Chebyshev collocation methods to Volterra integral equations with a weakly singular kernel, submitted to SIAM J. Numer. Anal.

[8] D. Colton And R. Kress, Inverse Acoustic and Electromagnetic Scattering Theory, Applied Mathematical Sciences 93, Springer-Verlag, Heidelberg, 2nd Edition (1998). MR.1635980 (99c:35181)

[9] T. Diogo, S. McKee, And T. TANG, Collocation methods for second-kind Volterra integral equations with weakly singular kernels, Proceedings of The Royal Society of Edinburgh, 124A, 1994, pp. 199-210. MR1273745 (95c:45011)

[10] A. Gogatishvili And J. LANG, The generalized Hardy operator with kernel and variable integral limits in Banach function spaces, Journal of Inequalities and Applications, 4 (1999), Issue 1, pp. 1-16. MR1733113 (2001f:47085)

[11] I. G. Graham and I. H. SloAn, Fully discrete spectral boundary integral methods for Helmholtz problems on smooth closed surfaces in $\mathbb{R}^{3}$, Numerische Mathematik, 92 (2002), pp. 289-323. MR1922922(2003h:65179)

[12] B. Guo, J. Shen AND L. Wang, Optimal spectral-Galerkin methods using generalized Jacobi polynomials, J. Sci. Comput. 27 (2006), 305-322. MR2285783 (2008f:65233)

[13] B. GUO AND L. WANG, Jacobi interpolation approximations and their applications to singular differential equations, Adv. Comput. Math. 14 (2001), pp. 227-276. MR.1845244(2002f:41003)

[14] B. Guo AND L. WANG, Jacobi approximations in non-uniformly Jacobi-weighted Sobolev spaces, J. Approx. Theory, 128 (2004), pp. 1-41. MR2063010 (2005h:41010)

[15] Q. Hu, Stieltjes derivatives and polynomial spline collocation for Volterra integro-differential equations with singularities. SIAM J. Numer. Anal., 33 (1996), 208-220. MR.1377251 (97a:65112)

[16] A. Kufner and L.E. Persson, Weighted Inequalities of Hardy Type, World Scientific, River Edge, NJ, 2003. MR1982932 (2004c:42034)

[17] Ch. Lubich, Fractional linear multi-step methods for Abel-Volterra integral equations of the second kind, Math. Comp., 45 (1985), pp. 463-469. MR804935(86j:65181)

[18] G. Mastroianni and D. Occorsio, Optimal systems of nodes for Lagrange interpolation on bounded intervals. A survey, Journal of Computational and Applied Mathematics, 134 (2001), pp. 325-341. MR.1852573 (2002e:65020)

[19] P. NevaI, Mean convergence of Lagrange interpolation. III, Trans. Amer. Math. Soc., 282 (1984), 669-698. MR85c:41009

[20] C. K. QU And R. Wong, Szegö's Conjecture on Lebesgue Constants for Legendre Series, Pacific J. Math., 135 (1988), pp. 157-188. MR965689 (89m:42025)

[21] D. L. RAGozin, Polynomial approximation on compact manifolds and homogeneous spaces, Trans. Amer. Math. Soc., 150 (1970), pp. 41-53. MR0410210(53:13960) 
[22] D. L. RAGozin, Constructive polynomial approximation on spheres and projective spaces, Trans. Amer. Math. Soc., 162 (1971), pp. 157-170. MR0288468 (44:5666)

[23] H. J.J. TE RIELE, Collocation methods for weakly singular second-kind Volterra integral equations with nonsmooth solution, IMA J. Numer. Anal., 2 (1982), pp. 437-449. MR692290 (84g:65167)

[24] S. G. Samko and R. P. Cardoso, Sonine integral equations of the first kind in $L_{p}(0, b)$, Fract. Calc. \& Appl. Anal. 2003, vol. 6, No 3, 235-258. MR2035650(2005a:45003)

[25] J. Shen And T. TAng, Spectral and High-Order Methods with Applications, Science Press, Beijing, 2006.

[26] T. TANG, Superconvergence of numerical solutions to weakly singular Volterra integrodifferential equations, Numer. Math., 61 (1992), pp. 373-382. MR1151776 (92k:65198)

[27] T. TANG, A note on collocation methods for Volterra integro-differential equations with weakly singular kernels, IMA J. Numer. Anal., 13 (1993), pp. 93-99. MR.1199031(93k:65111)

[28] T. TANG, X. Xu, AND J. Cheng, On spectral methods for Volterra integral equations and the convergence analysis, J. Comput. Math., 26 (2008), pp. 825-837. MR2464738

[29] T. TANG AND X. Xu, Accuracy enhancement using spectral postprocessing for differential equations and integral equations, Commun. Comput. Phys., 5 (2009), pp. 779-792.

[30] Z.-S. WAN, B.-Y. GUO AND Z.-Q. WANG, Jacobi pseudospectral method for fourth order problems, J. Comp. Math., 24 (2006), pp. 481-500. MR.2243117 (2007c:65063)

[31] D. Willett, A linear generalization of Gronwall's inequality, Proceedings of the American Mathematical Society, Vol. 16, No. 4. (Aug., 1965), pp. 774-778. MR0181726 (31:5953)

School of Mathematical Sciences, South China Normal University, Guangzhou 510631, CHINA

E-mail address: yanpingchen@scnu.edu.cn

Department of Mathematics, Hong Kong Baptist University, Kowloon Tong, Hong Kong - And- Faculty of Science, Beijing University of Aeronautics and Astronautics, BeIJING, China

E-mail address: ttang@math.hkbu.edu.hk 